\title{
Imitation of the impact of external factors in the modeling of innovation-oriented regional socio- economic systems
}

\author{
Tatyana Ladykova ${ }^{1, *}$, Inessa Vasilieva ${ }^{1}$ \\ ${ }^{1}$ Chuvash State University, 15, Moskovsky prospect, 428015, Cheboksary, Russia
}

\begin{abstract}
The regions of the Russian Federation function and develop in conditions of instability and uncertainty of the external strategic environment. Therefore, each region tries to develop optimal strategies for its innovation oriented development on the principles of efficiency and minimizing uncertainty (risk). At the same time, it is important not just to form a strategic set of alternatives for the development of the region, but it is important at every moment to know at what stage of development the region is, how close it is to the strategic goal of its development. Analysis of studies on strategic planning and management, shows the lack of a single accepted methodology for assessing the effectiveness of the implementation of regional strategies and general approaches to formation of system of indicators of its assessment. Nevertheless, the majority of researchers agree that it is important to assess not whether the implementation of the developed strategy is carried out correctly or not, but it is important to assess how close the implementation of the innovation oriented strategy is to the goals set for the region. Indicative planning is an important tool for regulating the processes of socio-economic development at the regional level. In modern conditions, the world economy is experiencing an increase in the level of negative impact of various external factors and major challenges to economic actors, including regions. The article substantiates the conceptual scheme of the imitation model of the regional socio-economic system, which takes into account not only the subjects and objects of management, but also the impact of the external environment. A method of increasing the realism of imitation models of the regional innovation oriented socio-economic system is proposed - the use of a random choice of one of the options for adaptation to a given external factor. Within the framework of this model, it is proposed to allocate the following macro-regional blocks (objects of regional management): income, labor, business, ecology, society, prospects, Finance. In this case, the control regulation within the framework of the proposed model is carried out on the basis of an indicative plan, which sets goals, objectives and indicators of regional innovation oriented socio-economic development.
\end{abstract}

\footnotetext{
* Corresponding author: ladykova@mail.ru
} 


\section{Introduction}

The influence of external factors on the functioning of regional innovation oriented socio-economic systems (RIOSES) leads to the need to develop new and adjust existing indicative planning mechanisms. The global economic crisis of 2008-2009 and the subsequent stagnation in conjunction with anti-Russian sanctions actualized the problem of ensuring the sustainability of regional development. Changes occur in the internal and external environment of the region, the frequency and amplitude of which are increasing, so the results of stress testing of economic models using a risk-based approach should be taken into account in the process of indicative planning.

In the conditions of negative external impact on the RIOSES, its stability can be ensured by the development of the innovative potential of enterprises of the basic circuit on the basis of operational monitoring of changes in the external environment [1]. Accordingly, simulation allows to reproduce these phenomena in computational experiments, and such analytical practice is actively used in the management of weakly structured systems [2].

However, currently existing mathematical models for objective reasons are not able to predict the sharp fluctuations of the environment with the necessary accuracy. The methodological basis for the development of negative scenarios are usually relatively "smooth" changes in the external environment, but in the conditions of" big challenges "the schedule of changes takes the form of a tortuous curve with a large number of" peaks", which are periodically transformed into "shocks". Hence, when adjusting development strategies during the computational experiment, exogenous variables, model parameters vary, its structure is improved, hypotheses about the behavior of individual parts of the system are refined. In addition, the construction of a realistic forecast of the development of the system is a costly process that requires the involvement of qualified specialists from different industries, since the imitation model takes into account both mathematical calculations and the logic of decision-making by the administration of the region. The state of RIOSES can be described by a variety of dynamic models [3, p. 51], and the choice of data is due only to the questions to be answered.

In General, it is impossible to limit the information base of the analysis, since any factor can have a decisive impact and suddenly appear on the planning horizon. This complexity is due to the variety of processes taking place in the region, the variant use of resources, the peculiarities of production cycles and the presence of links not only with different sectors of the economy, but also with social life. However, objective information does not always come to the developer of the model in a timely manner, and as a result, the correct decision is too late, and the price for the accuracy of the forecast becomes a decrease in the efficiency of system management.

\section{Applied methodology. Theoretical aspects of imitation modeling}

The emergence of imitation modeling was, on the one hand, due to the needs of practice, and on the other hand, the development of the method of statistical tests (Monte Carlo method), which opened the possibility to simulate random factors that significantly affect the functioning of the systems under consideration. To date, the theory, methods and technologies for creating different classes of models are well developed. Research in this branch of scientific knowledge continues with unabated intensity, covering more and more subject areas [4]. The main drawback of this approach was the fact that the simulation method over the decades has not become a mass tool. In addition, the introduction of the concept of "imitation" in science in the early 1960s was associated with a certain terminological confusion. The term simulation (imitation) modeling (from lat. simulation - 
likening) in the domestic literature is often translated as " imitation modeling " (it is enough to compare the original text and the Russian translation of the monograph by J. Forrester [5, 6]). At the same time, the terms "model" and "modeling" are used by specialists of various disciplines, as well as in everyday speech because of the universality of the concepts underlying the terms. Both concepts are closely related to what is today called a "system" or " process." Unfortunately, the conceptual apparatus here is not clearly defined, so scientists or engineers in discussions can use all these terms with the opposite meaning [3].

In contrast to the popular term "economic simulator" in the 2000 s, imitation modeling looks like a classic example of tautology ("simulation modeling"). In fact, the imitation model focuses on obtaining knowledge about the prototype not by analytical research or single numerical calculations, but by purposeful experiments $[4,8]$. While analytical modeling studies abstract (mathematical) models of a real object, simulation modeling evaluates mathematical models that reproduce the functioning of the analyzed system by performing a huge number of elementary operations [8]. The reliability of the conclusions thus depends on the adequacy of the constructed model, although formally the objectives of the simulation experiments may be different - from determining the properties and patterns of the analyzed system to perform specific tasks of indicative planning.

The economic simulator should reproduce both the structure of the object and its functioning with the necessary completeness, while maintaining the similarity of behavior in relation to the object. The type of model used depends not only on the system being investigated or designed, but also on the preference for the approach and choice of mathematical tools that will be used to successfully achieve the goal. Imitation is a computer experiment, and the difference between the experiment and real life is only that it is carried out with the model of the system, and not directly with the totality of socioeconomic relations that make up its essence and content.

It should also be borne in mind that imitation systems are characterized by greater proximity to the real system than mathematical models $[4,9,10,11]$. To a large extent, this proximity is due to the fact that the principles of construction of the imitation model make it possible to verify each block before its inclusion in the General model. "RIOSES simulator" may include complex dependencies that are not described by simple mathematical relations. Plausible simulation in a mathematical experiment can be achieved by introducing additional coefficients into the model, and then for modeling random factors it is necessary to know the degree of risk and the boundaries of the probabilistic action for individual blocks of the system. The rule by which random factors change is usually given by appropriate theoretical or empirical distribution functions. To do this, you need to use pseudo-random number generators to simulate the variability of certain events. However, boundless anti-determinism precludes independent verification of the stated hypotheses, since each time the model is tested, it will offer answers that differ quantitatively from each other.

In the formation of differential models, connections are established between the selected variables in the form of functions of the right parts of the equations of state. More productive is the approach based on a detailed description of the chains of cause-and-effect relationships (in modern modeling, the terms "storage-level" and "flow"are used). Levels describe the accumulation within the system and act as quantities that are considered as variables of the state of the system, describe quantities that are discrete in time but continuous over a range of values. In imitation modeling, the mathematical apparatus is extremely important, but it is used only at certain stages of creating a model. In addition, much of the success of the idea depends on the training and experience of researchers involved in the creation of the model. It is no secret that the modeling of the system is based on the knowledge of its properties that are important for a particular task. In this case, a system with certain properties can be represented in several ways and its mathematical 
model will not be uniquely defined, that is, it can have a different nature - for example, in the form of differential equations or describing functions [3].

The dominant trend today is the interpenetration of all types of modeling and synthesis of information technologies (especially for complex applications and complex projects). Modern software products do not require special training of the user, and he has the opportunity to pay more attention to the imitation experiments with the interpretation of the results. Perspective directions are imitation modeling of the system with its functioning [2, $7,12,13,14]$, as well as the display of real production in the form of a imitation model [9, $15,16,17]$.

Formalization of data using a new class of dynamic models allows to adequately describe the real behavior of the system, and to develop informed decisions on the management of the regional socio-economic system. However, in most cases, the imitation model is a multi-level software package based on systems of mathematical relations of equations and inequalities. The complexity and hidden problems in the class of modern continuous-discrete models require the development of new tools [11]. However, it should be noted that in the work with real projects, there is almost never data for correct statistical analysis.

The complexity of modeling is increasing and requires more computing power, but the innovative potential of research in this area is limited by external factors. For RIOSES models that operate in changing economic conditions, an open software system is needed whose properties are derived from the system itself, not from its structural components. The realism of the model increases if we consider the possibility of the system to rebuild the structure, to accumulate experience by automatically connecting to external information bases, to generate new data, to abandon inefficient solutions to the problem. In the classical approach, positive feedback will be manifested in changes in structural characteristics, and negative feedback - in the return to the previous stationary state after disturbances caused by external factors ("calls") [18]. The imitation model, taking into account the adaptability of the RIOSES, has the ability of its own transformation as a response to changes in the external environment. On the basis of such a system, it becomes possible to check the realism of regional socio-economic development strategies, as well as the consistency of goals and objectives in the framework of indicative plans [19].

\section{Results. Imitation model of RIOSES with a choice of random variants of adaptation to the influence of external factors}

A characteristic feature of the currently used the imitation modeling software is the focus on users-programmers, which creates significant difficulties for participation in the creation and operation of models of those users who do not have programming skills, but are specialists in their field. In this regard, it is important to study the existing models and develop problem-oriented packages based on them, which should provide an opportunity for specialists to independently create models and use them to solve various problems of analysis and synthesis of organizational and technical systems.

The concept of the RIOSES imitation model taking into account adaptation proceeds from the fact that the adaptation of economic entities to changes in the external environment affects the internal environment of the system [20]. As a rule, the stability index of regional development is expressed by an arithmetic mean calculated on the basis of matrices of sustainable development of territories [21]. In the region, it is possible to form the economic structure of industries and enterprises that are not related to each other, but ensure the stability of the region in case of external shocks due to the "case effect" [14]. In this case, the model of state strategic management of the economic sector, in which the element regulating the adaptability of the system is the level of uncertainty of the 
environment $(\mathrm{U})$, which can take values from 0 to 1 (minimum and maximum level of uncertainty), will be a model for building a imitation model of the RIOSES.

The value of the level of uncertainty, in turn, depends on the number of scenarios, strategy (q), the range of values of target indicators of the strategy (a), the timing of adjustment strategies $(\mathrm{t})$, the number of indicators for monitoring implementation of the strategy (i). The following dependence of the strategy on the $U$ level is justified; very high $\mathrm{U}$ (1-0.76) - maximum values of $\mathrm{q}, \mathrm{a}, \mathrm{i}$ and minimum $\mathrm{t}$, high $\mathrm{U}(0.75-0.51)$ - high values of $\mathrm{q}$, a, i and low t, average $\mathrm{U}(0.5-0.26)$ - low values of $\mathrm{q}, \mathrm{a}, \mathrm{i}$ and high $\mathrm{t}$, low $\mathrm{U}(0.25-0)$ minimum values of $q, a, i$ and maximum $t$ [15]. The block of imitation of industrial policy of RIOSES represents hierarchical two-level game where on the first level the state, and on the second - the concrete economic subject settles down [12]. In addition, it is necessary to take into account the results of the comparative analysis of plans for socio-economic development of the subject of the Russian Federation, territorial plans and monitoring of regional large-scale programs and projects $[21,22]$.

The realism of the model is enhanced by the use of neuromathematics. To predict the socio-economic development of Russian regions, a Bayesian ensemble of 5 neural networks in the form of multilayer perceptrons of different configurations is formed. The number of hidden layers ranges from 1 to 3, and the number of neurons in each of them - from 6 to 14 . The problem of approximation or reconstruction of hidden functional dependencies in the initial data is solved with the help of Bayesian ensemble of dynamic neural networks. In the process of constructing neural network models, it is required to create two arrays based on existing data, which are classified as training and testing. In this case, the training sample consists of observations distributed randomly in advance, and the testing sample consists of observations that make up an array of baseline indicators for all Russian regions for a particular year.

The proposed imitation model of RIOSES (Fig. 1) includes four main subsystems of the first level: the external environment, the region, the subject of regional management, the object of regional management (macro-regional unit). Since external change carries both threats and opportunities, in the first case, adaptation involves identifying problems, forecasting and planning them, as well as minimizing negative consequences, and in the second - maximizing the positive aspects of "threats and shocks" [1, 18, 23, 24].

Maximizing positive results for one of the actors may be perceived negatively for the system as a whole (for example, the reaction to an increase in fines for environmental violations may be the departure of business into the shadows or a reduction in production). In addition, a set of adequate options for responding to external changes can not be rigidly tied to quantitative indicators. As you know, using the Monte Carlo method it is possible to simulate not only the impact of certain factors on economic processes, but also the behavior of participants in transactions. This allows you to get rid of those difficulties that will inevitably arise when trying to use artificial intelligence algorithms to increase realism. 


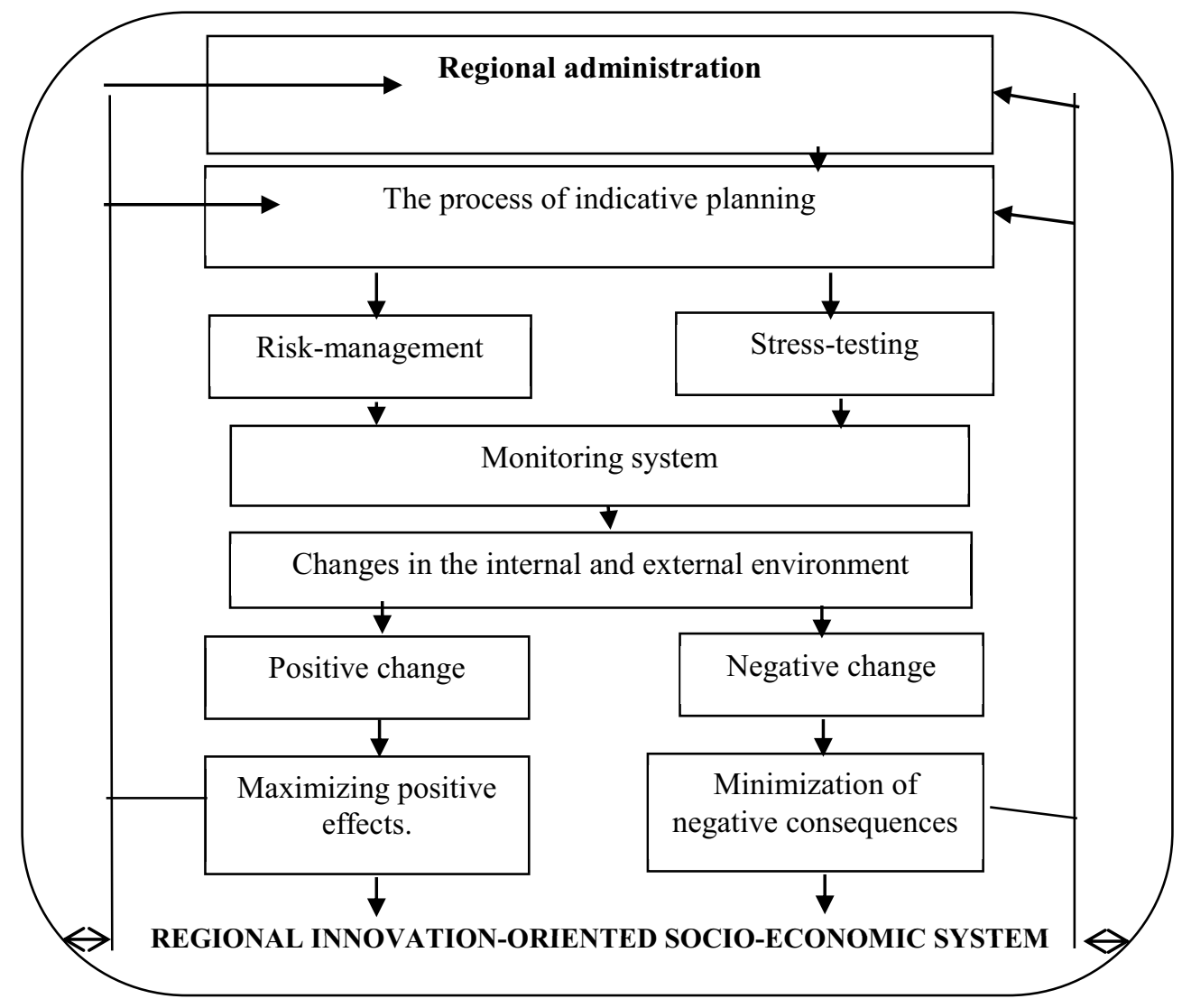

Fig. 1. Diagram of the imitation model of RIOSES.

Structurally, the proposed model can consist of three main levels: the external environment, the region, the subject of regional management. The external environment can be described as follows:

$$
E_{e}=F\left(P_{e}, R_{e}, S_{e}\right),
$$

where $P_{e}$ - the parameters of the environment, $R_{e}$ - the resources of the environment, $S_{e}$-scenarios for the development of the external environment.

The region in the framework of the proposed model can be represented through goal setting $\left(T_{r}\right)$, management $\left(M_{r}\right)$ and regional balance $\left(R B_{r}\right)$ :

$$
T_{r}=\left(I P_{r}, F L_{r}, I S_{r}\right),
$$

where $I P_{r}-$ the indicative plan of regional development, $F L_{r}$ - fuzzy logic,

$I S_{r}$ - the initial state of the regional economy,

$$
M_{r}=\left(I P_{r}, I S_{r}, S M_{r}, R M_{r}\right),
$$


where $I P_{r}$ - the indicative plan of regional development,

$I S_{r}$ - the initial state of the regional economy,

$S M_{r}$ - regional governance structures,

$R M_{r}$ - resources of regional governance structures,

$$
R B_{r}=\left(D_{r}, S_{r}\right)
$$

where $D_{r}$ - regional demand,

$S_{r}$ - regional proposal.

Due to the fact that the planning of socio-economic development is often used qualitative characteristics, such as the level or quality of life, it is advisable to use fuzzy logic algorithms [14]. The subject of regional management can be described by means of models of adaptive behavior $\left(A B_{s}\right)$, dynamics of resources $\left(R D_{s}\right)$, balance of the subject $\left(B S_{s}\right)$ :

$$
A B_{s}=\left(R_{s}, S_{s}, K_{s}, I S_{s}\right)
$$

where $R_{s^{-}}$resources possessed by the subject of regional management,

$S_{s}$ - strategy of the subject of regional management,

$K_{s}$ - class situation,

$I S_{s}$ - initial state of the subject of regional management,

$$
R D_{s}=\left(R R_{s}, R D_{s}\right) \text {, }
$$

where $R R_{s^{-}}$the amount of resources of the subject of management, $R D_{s}$ - dynamics of resources of the subject of management,

$$
B S_{s}=\left(P S_{s}, E S_{s}\right) \text {, }
$$

where $P S_{s}$ - income (receipts) of the subject, $E S_{s}$ - expenses (outflows) of the subject of management.

Within the framework of the proposed model, the following macro-regional blocks (objects of regional management) can be distinguished: income, labor, business, ecology, society, prospects, finance $[10,16,25,26,27]$. The functioning of each macro regional bloc can be described as follows:

$$
F O=\left(A B_{o}, R D_{o}, B S_{o}\right)
$$

where $A B_{o}$ - adaptive behavior of actors at the level of macro-regional block, $R D_{0}$ - dynamics of resources of the macro-regional unit, $B S_{\circ}$ - balance of the macro-regional bloc.

Each macro-regional block as a result of decomposition can be painted to take into account specific indicators [26]. For example, for the adaptive behavior block $A B_{S}(5)$, it would look like this: 


$$
A B_{s}=\left\{\begin{array}{c}
R_{s}=\left\{R_{s_{t}^{1}}, R_{s_{t}^{2}}, \ldots, R_{s_{t}^{n}}\right\} \\
S_{s}=\left\{S_{s_{t}^{1}}, S_{s_{t}^{2}}, \ldots, S_{s_{t}^{n}}\right\} \\
K_{s}=\left\{K_{s_{t}^{1}}, K_{s_{t}^{2}}, \ldots, K_{s_{t}^{n}}\right\} \\
I S_{s}=\left\{I S_{s_{t}^{1}}, I S_{s_{t}^{2}}, \ldots, I S_{s_{t}^{n}}\right\}
\end{array}\right.
$$

where $R_{s_{t}^{n}}, S_{s_{t}^{n}}, K_{s_{t}^{n}}, I S_{s_{t}^{n}} n-$ values, respectively, resources, strategy, situation classes and initial indicators of a particular macro-regional block.

In the formula (1):

$$
E_{e}=\left\{\begin{array}{l}
P_{e}=\left\{P_{e_{t}^{1}}, P_{e_{t}^{2}}, \ldots, P_{e_{t}^{n}}\right\} \\
R_{e}=\left\{R_{e_{t}^{1}}, R_{e_{t}^{2}}, \ldots, R_{e_{t}^{n}}\right\} \\
S_{e}=\left\{S_{e_{t}^{1}}, S_{e_{t}^{2}}, \ldots, S_{e_{t}^{n}}\right\}
\end{array}\right.
$$

In the formula (2):

$$
T_{r}=\left\{\begin{aligned}
I P_{r} & =\left\{I P_{r_{t}^{1}}, I P_{r_{t}^{2}}, \ldots, I P_{r_{t}^{n}}\right\} \\
F L_{r} & =\left\{F L_{r_{t}^{1}}, F L_{r_{t}^{2}}, \ldots, F L_{r_{t}^{n}}\right\} \\
I S_{r} & =\left\{I S_{r_{t}^{1}}, I S_{r_{t}^{2}}, \ldots, I S_{r_{t}^{n}}\right\}
\end{aligned}\right.
$$

In the formula (3):

$$
M_{r}=\left\{\begin{array}{c}
I P_{r}=\left\{I P_{r_{t}^{1}}, I P_{r_{t}^{2}}, \ldots, I P_{r_{t}^{n}}\right\} \\
I S_{r}=\left\{I S_{r_{t}^{1}}, I S_{r_{t}^{2}}, \ldots, I S_{r_{t}^{n}}\right\} \\
S M_{r}=\left\{S M_{r_{t}^{1}}, S M_{r_{t}^{2}}, \ldots, S M_{r_{t}^{n}}\right\} \\
R M_{r}=\left\{R M_{r_{t}^{1}}, R M_{r_{t}^{2}}, \ldots, R M_{r_{t}^{n}}\right\}
\end{array}\right.
$$

In the formula (4):

$$
R B_{r}=\left\{\begin{array}{c}
D_{r}=\left\{D_{r_{t}^{1}}, D_{r_{t}^{2}}, \ldots, D_{r_{t}^{n}}\right\} \\
S_{r}=\left\{S_{r_{t}^{1}}, S_{r_{t}^{2}}, \ldots, S_{r_{t}^{n}}\right\}
\end{array}\right.
$$

In the formula (6):

$$
R D_{s}=\left\{\begin{array}{l}
R R_{s}=\left\{R R_{s_{t}^{1}}, R R_{s_{t}^{2}}, \ldots, R R_{s_{t}^{n}}\right\} \\
R D_{s}=\left\{R D_{s_{t}^{1}}, R D_{s_{t}^{2}}, \ldots, R D_{s_{t}^{n}}\right\}
\end{array}\right.
$$

In the formula (7):

$$
B S_{s}=\left\{\begin{array}{l}
P S_{s}=\left\{P S_{s_{t}^{1}}, P S_{s_{t}^{2}}, \ldots, P S_{s_{t}^{n}}\right\} \\
E S_{s}=\left\{E S_{s_{t}^{1}}, E S_{s_{t}^{2}}, \ldots, E S_{s_{t}^{n}}\right\}
\end{array}\right.
$$

In the process of modeling, the $i$-th indicator of the indicative plan is sequentially determined taking into account internal and external factors at time $t$. This function also has a nested procedure for determining the $i j$-combination, where $j$ is a controlled parameter of the control structure $M_{r}$. In the process of monitoring, a decision is made to adjust the

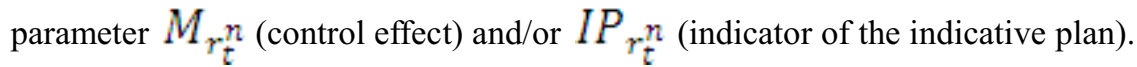




\section{Discussion}

Thus, in the framework of the presented model RIOSES is taken into account the following main groups of factors: external, specified in the "external environment" (1), management, concentrated in the block "control" (3), the parameters of the macro-regional block (8), plan presented in the "goal setting" (2). At the same time regional management can be carried out as follows:

- the probable scenarios of the external environment development are determined (1);

-the degree of influence of the external factor on the $i$-th indicator of the initial state of the regional economy (2) and (3);

- randomly selected one of the pre-formulated classes of the situation for a particular $i$ th indicator (5);

- depending on the selected class, the $i$-th index is saved, reduced, increased by a certain iterative value $\lambda_{t}^{i}$

- calculation of the model with new, adjusted indicators is carried out.

After reaching the last indicator of the corresponding class, the adjusted data serve as the basis for decision-making at the regional level.

\section{Conclusion}

In the modern conditions of increasing influence of external factors and big challenges on RIOSES, there is an objective need to develop new management tools. Taking into account the digitalization of all aspects of management activities in the process of indicative planning, it is necessary to use imitation modeling, which will improve the efficiency of indicative planning [27].

Very often, when building a model, accurate prediction of the quantitative characteristics of the system is not the main task. In order to identify General qualitative trends in the dynamics of variables and analyze the sensitivity of the results, it is most important to take proper account of the cause-and-effect relationships of the system. As a rule, the numerical results of the model with respect to the population conflict with the real data. This gives reason to doubt the correspondence of the model and reality, but this goal was not pursued by the authors of classical imitation models.

For example, the model J. Forrester was conceived as a simple educational example of the application of the method of system dynamics and became a model for subsequent work, drew attention to the problem. The model of $\mathrm{D}$. Meadows is much more detailed and seems more objective, but in comparison with the model of J. Forrester's is even more flawed. D. Meadows himself admitted that he had only $0.1 \%$ of the necessary information, that is, "the rest, apparently, he invented himself" [25]. However, even here the qualitative side of the results is more important, since the quantitative indicators are not so significant in comparison with the behavior of the system variables for imitation $[28,29,30]$.

The proposed method of computer experiment in the study of economic models makes it possible to form a control problem for the RIOSES simulator taking into account external factors. Based on the solution of this problem, practical recommendations can be developed to create a software package that simulates the existing decision support system in the regional economic system through the use of dynamic models in the study of real economic processes (both discrete and continuous). 


\section{Acknowledgements}

The work was prepared with the financial support of the RFBR in the framework of the scientific project No. 18-010-00459.

\section{References}

1. I.P. Danilov, T.I. Ladykova Oeconomia et Jus 1, 1 (2017)

2. A.V. Masloboev, A.G. Olejnik, M.G. Shishaev, Scientific and Technical Bulletin of Information Technologies, Mechanics and Optics 15(4), 748 (2015)

3. K.P. Bryanceva, O.V. Morgunova, Modern science: actual problems of theory and practice 1, 51 (2019)

4. A.M. Plotnikov, Yu.I. Ryzhikov, B.V. Sokolov, R.M. Yusupov, Conference proceedings «Simulation. Theory and practice» (IMMOD)). Edition SPIIRAN 2(25), 42 (2013)

5. J.W. Forrester, Industrial Dynamics. Portland (OR, Productivity Press, 1961)

6. J.W. Forrester, The Fundamentals of Enterprise Cybernetics (Industrial Dynamics) (Moscow, Progress, 1971)

7. E. Winsberg, Simulated Experiments: Methodology for a Virtual World. The University of Chicago Press. Philosophy of Science 70, 105 (2003)

8. N.I. Grishakina, A.S. Zareckaya, Modern technologies of decision-making in the digital economy: proceedings of the all-Russian scientific and practical conference of students, postgraduates and young scientists (Tomsk, 2018)

9. A.V. Mamatov, A.L. Mashkova, E.V. Novikova, O.A. Savina, Scientific and technical journal "Information systems and technologies» 2(112), 48 (2019)

10. S.V. Chuchkalov, Modeling of demographic processes in the Kirov region, Diss. on competition of a scientific degree (Perm)

11. N.A. Samsonova, Bulletin of the Central Economics and Mathematics Institute RAS 4 (2018) http://cemi.jes.su/s111111110000000-3-1/

12. V.V. Akberdina, A.V. Grebenkin, G.B. Korovin, A.I. Ponomareva, Russian Journal of the Economic Theory 15(4), 554 (2018)

13. K.A. Aksenov, E.M. Safrygina, L.G. Dorosinskij, Scientific and technical sheets of SPbSPU 1(115), 42 (2011)

14. S.N. Rastvorceva, Russian Journal of the Economic Theory 15(4), 554 (2018)

15. G.A. Lukashov, Public administration. E-Bulletin 47, 199 (2014)

16. A.A. Ponosov, Management of economic systems: electronic scientific journal 6(78) (2015) http://www.uecs.ru/

17. N.A. Semenov, Actual issues of economic Sciences. Proceedings of the XI International scientific and practical conference 3, 194 (2010)

18. I.L. Litvinenko, Scientific Bulletin of VF Ranepa 3, 34 (2015)

19. A.B. Hodzinskaya, Journal AGU 4(151), 118 (2014)

20. M.M. Nizamutdinov, V.V. Oreshnikov, Proceedings of the third international conference "Information technologies of intellectual decision support» (Ufa, 2015)

21. N.D. Kremlev, Russian Journal of the Economic Theory 15(2), 224 (2018)

22. N.G. Yushkova, Scientific statements of Belgorod State University. Series: Economics. 
Informatics 15-1(186), 14 (2014)

23. D. Mackinnon, A. Cumber, A. Pike at al., Economic Geography 85, 129 (2009)

24. B. Smit, J. Wandel, Global environmental change 16(3), 282 (2006)

25. T.I. Ladykova, V.L. Bersenev, Economy of Region 14(2), 380 (2018)

26. V. Holodkova, A. Mottaeva, T. Pokrovskaya, E3S Web of Conferences 164, 11043 (2020) https://doi.org/10.1051/e3sconf/202016411043

27. M. Wooldridge, N. Jennings, The Knowledge Engineering Review 10-2, 115 (1995)

28. D. Radushinsky, A. Mottaeva, The conference proceedings Energy Management of Municipal Transportation Facilities and Transport (EMMFT 2017) - IOP Conference Series: Earth and Environmental Science 90(1), 012137 (2017) DOI: https://doi.org/10.1088/1755-1315/90/1/012137

29. V. Krolivetskaya, E. Krolivetsky, E3S Web Conf. 135, 05026 (2019) DOI: https://doi.org/10.1051/e3sconf/201913504032

30. A. Evmenov, E. Krolivetsky, S. Krolivetskaya, T. Sorvina, E3S Web Conf. 164, 10036 (2020) DOI: https://doi.org/10.17323/j.jcfr.2073-0438.13.2.2019.104-113 